\title{
РЕГУЛЯРИЗОВАННЫЕ ПРОЕКЦИОННЫЕ МЕТОДЫ ДЛЯ НЕКОРРЕКТНЫХ ЗАДАЧ
}

\author{
(Представил А. Хумал)
}

\begin{abstract}
Исследуется регуляризация некорректных задач, дискретизированных проекционными методами. Даются условия, при которых размерность спроектированного уравнения является равноправным параметром регуляризации. Указаны правила выбора размерности и параметра регуляризации для получения регуляризующего алгоритма, а также для оптимальной точности его по порядку.
\end{abstract}

\section{1. Введение}

При решении некорректных задач применимы многие методы регуляризации $\left[{ }^{1-6}\right]$. Для реализации их на ЭВМ необходима дискретизация до или после регуляризации. Интерес представляет проблема выбора размерности $n$ в проекционном методе или шага $h$ в конечно-разностных методах.

Один подход к этому вопросу заключается в трактовке ошибок дискретизации как дополнительных возмущений оператора и правой части уравнения. Порядок регуляризации сохраняется при достаточно высоком $n$ или малом $h$. Так, в $\left[{ }^{7}\right]$ исследован выбор шага $h$ при решении методом конечных элементов уравнения, регуляризованного методом Тихонова. Использование проекционного метода Галеркина для уравнения, регуляризованного методом Лаврентьева, исследовано в $\left.{ }^{8}\right]$. Дискретизация уравнения проекционным методом наименьших квадратов и регуляризация ее затем методом Тихонова исследована в $\left[{ }^{9,10}\right]$, а также в $\left[{ }^{2}\right]$ (гл. 6 , $\S 6)$, эквивалентный алгоритм приведен в $\left[{ }^{11-13}\right]$. Выбор размерности при решении спроектированного уравнения методом Тихонова или итеращий проанализирован в $\left[{ }^{14}\right]$.

Другой подход возможен в случаях, когда решение дискретизированного первоначального уравнения сходится при $n \rightarrow \infty(h \rightarrow 0)$ к (нормальному) решению этого уравнения, если оператор и правая часть уравнения заданы точно. Такая сходимость для проекционных методов исследована в $\left[{ }^{9}\right]$ (теоремы $\left.(11)-(13)\right)$ и $\left[{ }^{15-23}\right]$, а для других методов в $\left[{ }^{6,24,25}\right]$ (см. и ссылки в них). Если в этом случае оператор и правая часть заданы неточно, то при правильном согласовании $n$ или $h$ с уровнями погрешностей получается регуляризующий алгоритм с параметром регуляризации $n$ или $h$ (иногда тогда говорят о саморегуляризации). В случае дополнительной регуляризации уравнения до или после дискретизации можно говорить о двухпараметрической регуляризации (см. $\left.\left[{ }^{26}\right]\right)$.

B $\left[{ }^{23}\right]$ установлены условия саморегуляризации при использовании некоторых проекционных методов. В настоящей работе исследуется выбор параметров при регуляризации соответствующих спроектированных уравнений любым методом из класса, выделенного в [ $\left.{ }^{5}\right]$ и вклю- 
чающего методы Тихонова, Лаврентьева, итерационные методы и прочие. С учетом эквивалентности некоторых методов (см. раздел 4) результаты разделов 3 и 4 используемы и при дискретизации регуляризованных уравнений.

\section{2. Задача и метод ее решения}

Пусть $H$ и $F-$ гильбертовы пространства и $A \in L(H, F)-$ вполне непрерывный оператор; нулевое пространство $N(A)$ в общем нетривиально. Рассмотрим уравнение

$$
A u=f .
$$

Введем ортопроекторы $P: H \rightarrow \overline{R\left(A^{*}\right)}, Q: F \rightarrow \overline{R(A)}$. Здесь $R(A)-$ область значений оператора $A, A^{*} \in L(F, H)-$ сопряженный с $A$ оператор. Предполагаем, что $f \in R(A)$. Пусть вместо $f$ и $A$ известны приближения $f_{\delta} \in F$ и $A_{\eta} \in L(H, F)$ такие, что $\left\|f_{\delta}-f\right\| \leqslant \delta$, $\left\|A_{\eta}-A\right\| \leqslant \eta$.

Опишем двухпараметрическую регуляризацию уравнения (1).

На первом этапе регуляризации используем проекционные методы [27]. Выбирая $n$-мерные подпространства $H_{n} \subset H$ и $F_{n} \subset F$ с соответствующими ортопроекторами $P_{n}$ и $Q_{n}$, получим спроектированное уравнение $Q_{n} A_{\eta} P_{n} u_{n}=Q_{n} f_{8}$ с искомым решением $u_{n} \in H_{n}$.

На втором этапе регуляризащии используем однопараметрическое семейство вещественнозначных функций $\left\{g_{\alpha}\right\}, \alpha>0$ (см. $\left[{ }^{5}\right]$ ), определенных и измеримых на некотором отрезке $\left[0, \lambda_{0}\right]$ и удовлетворяющих условиям

$$
\begin{array}{ll}
\sup _{0 \leqslant \lambda \leqslant \lambda_{0}}\left|g_{\alpha}(\lambda)\right| \leqslant C \alpha^{-1} & (\alpha>0), \\
\sup _{0 \leqslant \lambda \leqslant \lambda_{0}} \lambda^{p}\left|1-\lambda g_{\alpha}(\lambda)\right| \leqslant C \alpha^{p} & \left(0 \leqslant p \leqslant p_{0}, p_{0}>0\right) .
\end{array}
$$

Буквой $C$ здесь и в дальнейшем обозначим независящие от $\alpha ; n, \delta$ и $\eta$ постоянные, значения которых в разных местах в общем разные.

В случае $F=H, A=A^{*} \geqslant 0, A_{\eta}=A_{\eta}{ }^{*} \geqslant 0, F_{n}=H_{n}$ в качестве приближенного решения уравнения (1) примем элемент

$$
u_{n, \alpha}=\left(I-A_{\eta, n} g_{\alpha}\left(A_{\eta, n}\right)\right) P_{n} u_{0}+g_{\alpha}\left(A_{\eta, n}\right) P_{n} f_{\delta}, \quad A_{\eta, n}=P_{n} A_{\eta} P_{n},
$$

предполагая $\left\|A_{\eta, n}\right\| \leqslant \lambda_{0}$.

В случае несамосопряженной задачи (1) приближенное решение имеет вид

$$
\begin{aligned}
& u_{n, \alpha}=\left(I-B_{\eta, n} g_{\alpha}\left(B_{\eta, n}\right)\right) P_{n} u_{0}+g_{\alpha}\left(B_{\eta, n}\right) A_{\eta, n}^{*} f_{\delta}, \\
& A_{\eta, n}=Q_{n} A_{\eta} P_{n}, \quad B_{\eta, n}=A_{\eta, n}^{*} A_{\eta, n}=P_{n} A_{\eta}^{*} Q_{n} A_{\eta} P_{n} .
\end{aligned}
$$

Мы предполагали, что $\left\|B_{\eta, n}\right\| \leqslant \lambda_{0}$.

Здесь $u_{0}$ любое начальное приближение с условием $u_{0} \in \overline{R\left(A^{*}\right)}$; можно, в частности, положить $u_{0}=0$.

Отметим, что условие (3) в методе Тихонова и Лаврентьева выполнено с $p_{0}=1$, в явной и неявной итерационной схеме с $p_{0}=\infty$ (см. $\left.\left[{ }^{5}\right]\right)$.

\section{3. Выбор параметров}

Обозначим через $u_{*}$ решение уравнения (1), ближайшее к $u_{0}$. Ошибку $e_{n, \alpha}=\left\|u_{n, \alpha}-u_{*}\right\|$ оцениваем на классе 
$u_{0}-u_{*}=|A|^{p} v, \quad u_{0}=|A|^{p} w ; \quad p>0 ; \quad v, w \in H,\|v\| \leqslant \mathrm{Q},\|w\| \leqslant \mathrm{Q}$,

где $|A|=\left(A^{*} A\right)^{1 / 2}$.

3 а м е ч ан и е 1 . Требование о представимости $u_{0}$ в виде $u_{0}=|A|^{p}$ w второстепенное, так как при $u_{0} \neq 0$ с заменой переменных $\bar{u}=u_{*}-u_{0}$ в уравнении (1) приходим к задаче $A \bar{u}=f-A u_{0}$, где начальному приближению $u_{0}$ соответствует $\bar{u}_{0}=0$.

Известно $\left(\left[{ }^{2,5}\right]\right)$, что оптимальной по порядку оценкой на классе $(6)$ является

$$
e_{n,:} \leqslant C \Delta^{p /(p+1)}, \quad \Delta=\max \{\delta, \eta\} .
$$

Обозначим $h=h(n)=\left\|\left(Q_{n} A P_{n}\right)^{-1} Q_{n}\right\|^{-1}$. В дальнейшем будут использованы условия вида

$$
\begin{aligned}
\left\|\left(I-P_{n}\right)|A|^{p}\right\| \leqslant C h^{p} & \left(0 \leqslant p \leqslant p_{1}\right), \\
\left\|\left(I-Q_{n}\right)\left|A^{*}\right|^{p}\right\| \leqslant C h^{p} & \left(0 \leqslant p \leqslant p_{2}\right) .
\end{aligned}
$$

Обозначим $a \asymp b$, если $a=O(b)$ и $b=O(a)$.

Теорем а 1 . Пусть $F=H, A=A^{*} \geqslant 0, A_{\eta}=A_{\eta}{ }^{*} \geqslant 0$ и уравнение (1) проектируется методом Галеркина (т. е. $F_{n}=H_{n} ;$ тогда (см. $\left.\left[{ }^{23}\right]\right)$ $\left.h=\inf \left\{\left(A u_{n}, u_{n}\right) /\left\|u_{n}\right\|^{2}, \quad u_{n} \in H_{n}\right\}\right)$. Пусть $H_{n} \cap N(A)=0, P_{n} u \rightarrow u$ при $n \rightarrow \infty \forall u \in \overline{R\left(A^{*}\right)}$. Предполагаем, что выполнено условие (8) $c$ $p_{1} \geqslant 1 / 2$.

Если в приближении (4) параметры $n(h)$ u а выбирать так, что $n \rightarrow \infty, \alpha \rightarrow 0, \Delta /(h+\alpha) \rightarrow 0$ nрu $\Delta \rightarrow 0$, то $e_{n, \alpha} \rightarrow 0$ nрu $\Delta \rightarrow 0$.

При предположении (6) справедлива оценка (7), если выбор параметров $n(h)$ и а подчинен условиям

1) $h+\alpha \asymp \Delta^{1 /(p+1)} \quad$ nрu $p \leqslant p_{0}, \quad p \leqslant p_{1}$;

2) $h \asymp \Delta^{1 /(p+1)}, \quad \alpha=O\left(\Delta^{p /\left(p_{0}(p+1)\right)}\right) \quad$ npu $\quad p_{0}<p \leqslant p_{1}$;

3) $\alpha \asymp \Delta^{1 /(p+1)}, \quad h=O\left(\Delta^{p /\left(p_{1}(p+1)\right)}\right) \quad$ при $\quad p_{1}<p \leqslant p_{0}$.

Т е о р е м 2. Пусть уравнение (1) проектируется методом наименьших квадратов (т. е. $F_{n}=A_{\eta} H_{n} ;$ тогда (см. $\left.\left[{ }^{23}\right]\right) \quad h=\inf \left\{\left\|A u_{n}\right\| /\left\|u_{n}\right\|\right.$, $\left.\left.u_{n} \in H_{n}\right\}\right)$. Пусть $H_{n} \cap N(A)=0, P_{n} u \rightarrow u$ nрu $n \rightarrow \infty \quad \forall u \in \overline{R\left(A^{*}\right)}$. Предполагаем, что условие (8) выполнено с $p_{1} \geqslant 1$.

Если в приближении (5) параметры $n(h)$ и а выбирать так, что $n \rightarrow \infty, \alpha \rightarrow 0, \Delta /\left(h+\alpha^{1 / 2}\right) \rightarrow 0$ nрu. $\Delta \rightarrow 0$, то $e_{n, \alpha} \rightarrow 0$ при $\Delta \rightarrow 0$.

При предположении (6) справедлива оценка (7), если выбор параметров $n(h)$ и а подчинен условиям

1) $h+\alpha^{1 / 2} \asymp \Delta^{1 /(p+1)} \quad$ npu $p \leqslant 2 p_{0}, \quad p \leqslant p_{1}$;

2) $h \asymp \Delta^{1 /(p+1)}, \quad \alpha=O\left(\Delta^{p /\left(p_{0}(p+1)\right)}\right) \quad$ npu $\quad 2 p_{0}<p \leqslant p_{1}$;

3) $\alpha \asymp \Delta^{2 /(p+1)}, \quad h=O\left(\Delta^{p /\left(p_{1}(p+1)\right)}\right) \quad$ npu $\quad p_{1}<p \leqslant 2 p_{0}$.

Т ео ре м а 3. Пусть уравнение (1) проектируется методом наименьшей ошибки (т. е. $H_{n}=A_{\eta}{ }^{*} F_{n} ;$ тогда (см. $\left.\left.{ }^{23}\right]\right) \quad h=\inf \left\{\left\|A^{*} f_{n}\right\| /\left\|f_{n}\right\|\right.$, $\left.\left.f_{n} \in F_{n}\right\}\right)$. Пусть $F_{n} \cap N\left(A^{*}\right)=0, Q_{n} f \rightarrow f$ nрu $\left.n \rightarrow \infty \quad \forall f \in \overline{R(A}\right)$.

Если в приближении (5) параметры $n(h) u$ a выбирать так, что $n \rightarrow \infty, \alpha \rightarrow 0, \Delta /\left(h+\alpha^{1 / 2}\right) \rightarrow 0$ nри $\Delta \rightarrow 0$, то $e_{n, \alpha} \rightarrow 0$ при $\Delta \rightarrow 0$.

При предположении (6) справедлива оценка (7), если выполнено условие (9) с $p_{2} \geqslant 1$ и выбор параметров $n(h) u$ а подчинен условиям

1) $h+\alpha^{1 / 2} \asymp \Delta^{1 /(p+1)} \quad$ npu $p \leqslant 2 p_{0}, \quad p \leqslant p_{2}+1$;

2) $h \asymp \Delta^{1 /(p+1)}, \quad \alpha=O\left(\Delta^{p /\left(p_{0}(p+1)\right)}\right) \quad$ npu $\quad 2 p_{0}<p \leqslant p_{2}+1$;

3) $\alpha \asymp \Delta^{2 /(p+1)}, \quad h=O\left(\Delta^{p /\left(\left(p_{2}+1\right)(p+1)\right)}\right) \quad$ npu $\quad p_{2}+1<p \leqslant 2 p_{0}$. 
Теоремы $1-3$ доказаны в разделе 7 .

Главный интерес в теоремах представляет случай 1), в котором параметры $h$ и $\alpha$ выступают как равноправные. В случаях 2) и 3) один из параметров следует подчинить другому. Ряд дополнений к теоремам приведем в виде замечаний. Первое из них касается любых проекционных методов. Обозначим $h_{\eta}=\left\|\left(Q_{n} A_{\eta} P_{n}\right)^{-1} Q_{n}\right\|^{-1}$.

3 ам еч ание 1 . Пусть $f \in R(A)$ и $P_{n} u_{*} \rightarrow u_{*},\left\|Q_{n} A P_{n}-A\right\| \rightarrow 0$ при $n \rightarrow \infty$. Если в приближении (5) выбор параметров $n(h)$ и $\alpha$ подчинен условию $\Delta+\left\|Q_{n} A\left(I-P_{n}\right) u_{*}\right\|=o\left(\alpha^{1 / 2}+h_{\eta}\right)$, то $e_{n \alpha} \rightarrow 0$ при $\Delta \rightarrow 0$. C учетом неравенства $\left\|Q_{n} A\left(I-P_{n}\right) u_{*}\right\| \leqslant\left\|\left(I-P_{n}\right) A^{*}\right\|\left\|\left(I-P_{n}\right) u_{*}\right\|$ это замечание усиливает результаты $\left[{ }^{10-13}\right]$.

3 а мечани е 2. Сходимость $e_{n, \alpha} \rightarrow 0$ при $\Delta \rightarrow 0$ в теореме 1 остается в силе при замене требования $p_{1} \geqslant 1 / 2$ дополнительным условием на выбор параметров: требуем $\left\|A^{1 / 2}\left(I-P_{n}\right) u_{\star}\right\| /(\alpha+h)^{1 / 2} \rightarrow 0$ при $\Delta \rightarrow 0$. 3 а мечание 3 . Условия (8), (9) достаточно проверить при $p=p_{1}$, $p=p_{2}$ соответственно, так как по неравенству моментов (см. $\left.\left[{ }^{27}\right]\right)$

$$
\begin{array}{rll}
\left\||A|^{p}\left(I-P_{n}\right)\right\| \leqslant\left\||A|^{p_{1}}\left(I-P_{n}\right)\right\| p / p_{1} & \text { при } & p \leqslant p_{1} ; \\
\left\|\left|A^{*}\right|^{p}\left(I-Q_{n}\right)\right\| \leqslant\left\|\left|A^{*}\right|^{p_{2}}\left(I-Q_{n}\right)\right\|^{p / p_{2}} & \text { при } & p \leqslant p_{2} .
\end{array}
$$

3 амечание 4 . В выражениях $h+\alpha$ в теореме 1 и в выражениях $h+\alpha^{1 / 2}$ в теоремах 2,3 величина $h$ может быть заменена величиной $h_{\eta}$. Эквивалентность выражений при $\Delta \rightarrow 0$ вытекает из предположений $\eta /(h+\alpha) \rightarrow 0$ (теорема 1$), \eta /\left(h+\alpha^{1 / 2}\right) \rightarrow 0$ (теоремы 2,3$)$ при $\Delta \rightarrow 0$ и из оценок $h-\eta \leqslant h_{\eta} \leqslant h+\eta$ при условии $\eta<h$ (см. $\left.\left[{ }^{23}\right]\right)$.

\section{4. О числе обусловленности при регуляризации спроектированных уравнений методом Лаврентьева или Тихонова}

Отметим сперва эквивалентность некоторых алгоритмов. Пусть $F=H$, $A=A^{*} \geqslant 0, A_{\eta}=A_{\eta}{ }^{*} \geqslant 0$. Если уравнение (1) дискретизуется методом Галеркина $\mathbf{n}$ регуляризуется затем методом Лаврентьева, то приближение $u_{n, \alpha}$ находится из уравнения

$$
\left(\alpha I+P_{n} A_{\eta} P_{n}\right) u_{n, \alpha}=P_{n} f_{\delta} .
$$

Такое же уравнение получается при регуляризации уравнения (1) методом Лаврентьева и дискретизации затем методом Галеркина.

При дискретизации уравнения (1) методом наименьших квадратов и регуляризации затем методом Тихонова получается уравнение

$$
\left(\alpha I+P_{n} A_{\eta}^{*} A_{\eta} P_{n}\right) u_{n, \alpha}=P_{n} A_{\eta}^{*} f_{\delta}
$$

возникающее также после применения методов Тихонова и Галеркина K (1).

В случае использования методов наименьшей ошибки и Тихонова уравнение (1) принимает вид $\left(\alpha I+A_{\eta}{ }^{*} Q_{n} A_{\eta}\right) u_{n, \alpha}=A_{\eta}{ }^{*} Q_{n} f_{\delta}$. Другой вариант реализации методов состоит в нахождении приближения $f_{n, \alpha}$ из уравнений

$$
\left(\alpha I+Q_{n} A_{\eta} A_{\eta}^{*} Q_{n}\right) f_{n, \alpha}=Q_{n} f_{\delta}
$$

а потом $u_{n, \alpha}=A_{\eta}{ }^{*} f_{n, \alpha}$.

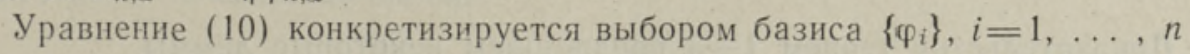
в $H_{n}$. Коэффициенты $x_{i}, i=1, \ldots, n$ в представлении $u_{n, \alpha}=\sum_{i=1}^{n} x_{i} \varphi_{i}$ находятся из системы линейных уравнений $B_{n} \mathbf{x}=\mathbf{b}$ с $\left(B_{n}\right)_{i j}=$ 
$=\left(\left(\alpha I+A_{\eta}\right) \varphi_{i}, \varphi_{j}\right), \quad b_{j}=\left(f_{\delta}, \varphi_{j}\right), i, j=1, \ldots, n$. Оценим число обусловленности матрицы $B_{n}$ - отношение максимального и минимального собственного значения. Воспользуемся следующим результатом из $\left[{ }^{23}\right]$.

Л е м м а 1. При любом операторе $B \in L(H, H), B=B^{*} \geqslant 0$ для числа обусловленности $k\left(B_{n}\right)$ матрицы $B_{n}$ с элементами $\left(B_{n}\right)_{i j}=\left(B \varphi_{i}, \varphi_{j}\right)$ справедлива оценка

$$
k\left(B_{n}\right) \leqslant\|B\| k\left(D_{n}\right) / v_{n}, \quad v_{n}=\inf \left\{\left(B u_{n}, u_{n}\right) /\left\|u_{n}\right\|^{2}, u_{n} \in H_{n}\right\},
$$

где $k\left(D_{n}\right)$ число обусловленности матрицы Грама $D_{n},\left(D_{n}\right)_{i j}=\left(\varphi_{i}, \varphi_{j}\right)$.

Для уравнения (10) отсюда при $B=\alpha I+A_{\eta}$ имеем $v_{n}=\alpha+h_{\eta}$,

$$
k\left(B_{n}\right) \leqslant(\alpha+\eta+\|A\|) k\left(D_{n}\right)\left(\alpha+h_{\eta}\right)^{-1} .
$$

Уравнение (11) также равносильно системе линейных уравнений $B_{n} \mathbf{X}=\mathbf{b}$, здесь $\left(B_{n}\right)_{i j}=\alpha\left(\varphi_{i}, \varphi_{j}\right)+\left(A_{\eta} \varphi_{i}, A_{\eta} \varphi_{j}\right), b_{j}=\left(f_{\delta}, A_{\eta} \varphi_{j}\right)$. Из леммы 1 при $B=\alpha I+A_{\eta}{ }^{*} A_{\eta}$ имеем $v_{n}=\alpha+h_{\eta}{ }^{2}$ и

$$
k\left(B_{n}\right) \leqslant\left(\alpha+\eta+\|A\|^{2}\right) k\left(D_{n}\right)\left(\alpha+h_{\eta}^{2}\right)^{-1} .
$$

Пусть решение уравнения (12) ищется в виде $f_{n, \alpha}=\sum_{i=1}^{n} x_{i} \psi_{i}$, где $\left\{\psi_{i}\right\}, i=1, \ldots, n-$ базис $F_{n}$ с матрицей Грама $\bar{D}_{n},\left(\bar{D}_{n}\right)_{i j}=\left(\psi_{i}, \psi_{j}\right)$. Вектор коэффициентов $\mathbf{x}$ находится из уравнений $B_{n} \mathbf{x}=\mathbf{b}$, где $\left(B_{n}\right)_{i j}=\alpha\left(\psi_{i}, \psi_{j}\right)+\left(A_{\eta}{ }^{*} \psi_{i}, A_{\eta}{ }^{*} \psi_{j}\right), \quad b_{j}=\left(f_{\delta}, \psi_{j}\right) . \quad$ Заменяя в лемме 1 $\varphi_{i}$ на $\psi_{i}, B$ на $\alpha I+A_{\eta} A_{\eta}{ }^{*}, u_{n}$ на $f_{n} ;$ имеем $\bar{v}_{n}=\inf \left\{\left(\left(\alpha I+A_{\eta} A_{\eta}{ }^{*}\right) f_{n}, f_{n}\right) /\right.$ $\left./\left\|f_{n}\right\|^{2}, f_{n} \in F_{n}\right\}=\alpha+h_{\eta}^{2}$ и

$$
k\left(B_{n}\right) \leqslant\left(\alpha+\eta+\|A\|^{2}\right) k\left(\bar{D}_{n}\right)\left(\alpha+h_{\eta}^{2}\right)^{-1} .
$$

Отметим, что базисы $\left\{\varphi_{i}\right\},\left\{\psi_{i}\right\}$ можно выбирать так, что $k\left(D_{n}\right) \leqslant C$, $k\left(\bar{D}_{n}\right) \leqslant C$.

\section{5. Приложения к интегральному уравнению Вольтерра I рода}

Рассмотрим интегральное уравнение Вольтерра I рода

$$
\int_{0}^{t} K(t, s) u(s) d s=f(t)
$$

как операторное уравнение $A u=f$ с $A: L_{2}[0,1] \rightarrow L_{2}[0,1]$. Пусть ядро представимо в виде $K(t, s)=H(t, s)(t-s)^{l-1}, \quad$ где $H(t, t)>0$, $0 \leqslant t \leqslant 1$, а $l-$ положительное целое число. Предполагаем, что при $i=1, \ldots, l$ величины $\frac{\partial^{i} H}{\partial t^{i}}(t-s)^{i-1}, \frac{\partial^{i} H}{\partial s^{i}}(s-t)^{i-1}$ ограничены. Обозначим через $S_{n}{ }^{k}$ подпространство сплайнов на $[0,1]$ порядка $k-1$ с узлами $t_{i}=i / n, \quad i=0, \ldots, n$. Из результатов $\left[{ }^{23}\right]$ вытекает, что в методе наименьших квадратов для (13) с $H_{n}=S_{n}{ }^{k}$ условие (8) выполнено с $p_{1}=k / l$, в методе наименьшей ошибки для (13) с $F_{n}=S_{n}{ }^{k}$ условие (9) выполнено с $p_{2}=k / l$; в обоих случаях $h \geqslant C n^{-l}$. В действительности $h \asymp n^{-l}$. Именно, при выборе $u_{n} \in S_{n}{ }^{k}$ и $f_{n} \in S_{n}{ }^{k}$ так, что $u_{n}(t)=0$ при $t \notin(1-1 / n, 1), f_{n}(t)=0$ при $t \notin(0,1 / n)$, легко оценить $\left\|A u_{n}\right\| /\left\|u_{n}\right\| \leqslant C n^{-l},\left\|A^{*} f_{n}\right\| /\left\|f_{n}\right\| \leqslant C n^{-l}$.

\section{6. Вспомогательные результаты}

Доказательству теорем $1-3$ предпошлем некоторые леммы.

Лемм а 2 (см. $\left.\left[{ }^{5}\right]\right)$. При $B, B_{\eta} \in L(H, F),\left\|B_{\eta}-B\right\| \leqslant \eta u$ любом вещественном $r \geqslant 0$ справедлива оценка

$$
\left\|\left|B_{\eta}\right|^{r}-|B|^{r}\right\| \leqslant C(1+|\ln \eta|) \eta^{\min (1, r)},
$$


при $B=B^{*} \geqslant 0, B_{\eta}=B_{\eta}{ }^{*} \geqslant 0$ справедлива также

$$
\left\|B_{\eta}^{r}-B^{r}\right\| \leqslant C \eta^{\min (1, r)} \text {. }
$$

Л ем м а 3. При $F=H, A=A^{*} \geqslant 0, A_{\eta}=A_{\eta}{ }^{*} \geqslant 0, F_{n}=H_{n}$ справедливы оценки

$$
\begin{gathered}
\left\|\left(I-P_{n}\right) A_{\eta}^{r}\right\| \leqslant C\left(\eta^{\min (r, 1)}+h^{\min \left(r, p_{1}\right)}\right), \\
\left\|g_{\alpha}\left(A_{\eta, n}\right) P_{n}\right\| \leqslant C\left(\alpha+h_{\eta}\right)^{-1}, \\
\left\|g_{\alpha}\left(A_{\eta, n}\right) P_{n} A_{\eta}\left(I-P_{n}\right)\right\| \leqslant C\left(\eta^{1 / 2}+h^{\min \left(1 / 2, p_{1}\right)}\right) /\left(\alpha+h_{\eta}\right)^{1 / 2},
\end{gathered}
$$

әде $г$ любое вещественное число и $A_{\eta, n}=P_{n} A_{\eta} P_{n}$.

Д о к а з а т е л ь с т в о. Оценка (16) вытекает из (15), (8) и неравенства $\left\|\left(I-P_{n}\right) A_{\eta}{ }^{r}\right\| \leqslant\left\|I-P_{n}\right\|\left\|A_{\eta}{ }^{r}-A^{r}\right\|+\left\|\left(I-P_{n}\right) A^{r}\right\|$. По условиям (2), (3) имеем $\left\|g_{\alpha}\left(A_{\eta, n}\right) P_{n}\right\| \leqslant\left\|g_{\alpha}\left(A_{\eta, n}\right)\right\| \leqslant C \alpha^{-1}, \quad\left\|g_{\alpha}\left(A_{\eta, n}\right) A_{\eta, n}\right\| \leqslant 1+$ $+\left\|I-g_{\alpha}\left(A_{\eta, n}\right) A_{\eta, n}\right\| \leqslant C$. C помощью неравенств $\left\|g_{\alpha}\left(A_{\eta, n}\right) P_{n}\right\| \leqslant$ $\leqslant\left\|g_{\alpha}\left(A_{\eta, n}\right) A_{\eta, n}\right\|\left\|A_{\eta, n}^{-1} P_{n}\right\| \leqslant C h_{\eta}^{-1}, \quad \min \left(\alpha^{-1}, h_{\eta}^{-1}\right) \leqslant 2\left(\alpha+h_{\eta}\right)^{-1}$ получаем оценку (17). Оценка (18) вытекает из (16), (17) и соотношений

$$
\begin{aligned}
& \left\|g_{\alpha}\left(A_{\eta, n}\right) P_{n} A_{\eta}\left(I-P_{n}\right)\right\| \leqslant\left\|g_{\alpha}\left(A_{\eta, n}\right) P_{n} A_{\eta}^{1 / 2}\right\|\left\|A_{\eta}^{1 / 2}\left(I-P_{n}\right)\right\|, \\
& \left\|g_{\alpha}\left(A_{\eta, n}\right) P_{\eta} A_{\eta}^{1 / 2}\right\|=\left\|g_{\alpha}\left(A_{\eta, n}\right) P_{n} A_{\eta}^{1 / 2}\left[g_{\alpha}\left(A_{\eta, n}\right) P_{n} A_{\eta}^{1 / 2}\right]^{*}\right\|^{1 / 2}= \\
& =\left\|g_{\alpha}\left(A_{\eta, n}\right) A_{\eta, n} P_{n} g_{\alpha}=\left(A_{\eta, n}\right)\right\|^{1 / 2} \leqslant C\left\|P_{n} g_{\alpha}\left(A_{\eta, n}\right)\right\|^{1 / 2} .
\end{aligned}
$$

Л ем м а 4. Справедливы неравенства

$$
\begin{gathered}
\left\|g_{\alpha}\left(B_{\eta, n}\right) A_{\eta, n}^{*}\right\| \leqslant C\left(\alpha^{1 / 2}+h_{\eta}\right)^{-1} ; \\
\left\|g_{\alpha}\left(B_{\eta, n}\right) A_{\eta, n}^{*} A_{\eta}\left(I-P_{n}\right)\right\| \leqslant C\left(\eta+\hbar^{\min \left(1, p_{1}\right)}\right) /\left(\alpha^{1 / 2}+h_{\eta}\right) ;
\end{gathered}
$$

где $A_{\eta, n}=Q_{n} A_{\eta} P_{n}, \quad B_{\eta, n}=A_{\eta, n}^{*} A_{\eta, n}$.

Д ок а з а тел ь с т в о. По условиям (2), (3) имеем $\left\|g_{\alpha}\left(B_{\eta, n}\right)\right\| \leqslant C \alpha^{-1}$, $\left\|g_{\alpha}\left(B_{\eta, n}\right) B_{\eta, n}\right\| \leqslant C . \quad$ Далее, $\quad\left\|g_{\alpha}\left(B_{\eta, n}\right) A_{\eta, n}^{*}\right\|^{2}=\| g_{\alpha}\left(B_{\eta, n}\right) A_{\eta, n}^{*} \times$ $\times\left[g_{\alpha}\left(B_{\eta, n}\right) A_{\eta, n}^{*}\right]^{*}\|=\| g_{\alpha}\left(B_{\eta, n}\right) B_{\eta, n} g_{\alpha}\left(B_{\eta, n}\right)\|\leqslant\| g_{\alpha}\left(B_{\eta, n}\right) B_{\eta, n} \| \times$ $X\left\|g_{\alpha}\left(B_{\eta, n}\right)\right\| \leqslant C \alpha^{-1}$. Учитывая равенство $\left(B_{\eta, n}\right)^{-1} A_{\eta, n}^{*}=A_{\eta, n}^{-1} Q_{n}$, получим $\left\|g_{\alpha}\left(B_{\eta, n}\right) A_{\eta, n}^{*}\right\| \leqslant\left\|g_{\alpha}\left(B_{\eta, n}\right) B_{\eta, n}\right\|\left\|\left(B_{\eta, n}\right)^{-1} A_{\eta, n}^{*}\right\| \leqslant C h_{\eta}^{-1}$. Оценка (19) вытекает теперь из неравенства $\min \left(\alpha^{-1 / 2}, h_{\eta}{ }^{-1}\right) \leqslant 2\left(\alpha^{1 / 2}+h_{\eta}\right)^{-1}$. Оценка (20) имеет место в силу (19), (8) и неравенств $\left\|g_{\alpha}\left(B_{\eta, n}\right) A_{\eta, n}^{*} A_{\eta}\left(I-P_{n}\right)\right\| \leqslant\left\|g_{\alpha}\left(B_{\eta, n}\right) A_{\eta, n}^{*}\right\| \quad\left(\left\|A\left(I-P_{n}\right)\right\| \neq \eta\right)$, $\left\|A\left(I-P_{n}\right)\right\| \leqslant\left\||A|\left(I-P_{n}\right)\right\|$. Последнее неравенство следует из полярного разложения (см. напр., $\left[{ }^{5}\right]$, с. 21) $A=U|A|$, где $U$ - частичная изометрия, $\|U\|=1$.

Л ем м а 5. Если условие (9) выполнено с $p_{2} \geqslant 1$, то при $H_{n}=A_{\eta}{ }^{*} F_{n}$ справедлива оценка

$$
\left\|\left(I-P_{n}\right)|A|^{p}\right\| \leqslant C h^{1+\min \left(p-1, p_{2}\right)}+C(1+|\ln \eta|) \eta^{\min (p, 1)} .
$$

Док аз ательство. По (14) имеем

$$
\left\|\left(I-P_{n}\right)|A|^{p}\right\| \leqslant\left\|\left(I-P_{n}\right)\left|A_{\eta}\right|^{p}\right\|+C(1+|\ln \eta|) \eta^{\min (p, 1)} .
$$

При $p \geqslant 2$ справедлива оценка

$$
\left\|\left(I-P_{n}\right)\left|A_{\eta}\right|^{p}\right\| \leqslant\left\|A_{\eta}^{*}\left(I-Q_{n}\right) A_{\eta}\left|A_{\eta}\right|^{p-2}\right\|,
$$

так как по свойству $H_{n}=A_{\eta}{ }^{*} F_{n}$ имеем $\left\|\left(I-P_{n}\right)\left|A_{\eta}\right|^{p v}\right\|=$ $=\inf \left\{\left\|\left|A_{\eta}\right|^{p} v-A_{\eta}^{*} f_{n}\right\|, \quad f_{n} \in F_{n}\right\} \leqslant\left\|A_{\eta}^{*} A_{\eta}\left|A_{\eta}\right|^{p-2} v-A_{\eta}^{*} Q_{n} A_{\eta}\left|A_{\eta}\right|^{p-2} v\right\|$. 
Убедимся в справедливости оценок

$$
\begin{gathered}
\left\|A_{\eta}^{*}\left(I-Q_{n}\right)\right\| \leqslant C(h+\eta), \\
\left\|A_{\eta}^{*}\left(I-Q_{n}\right) A_{\eta}\right\| \leqslant C(h+\eta)^{2} .
\end{gathered}
$$

Воспользуясь полярным разложением $A^{*}=U^{*}\left|A^{*}\right|,\left\|U^{*}\right\|=1$, имеем $\left\|A_{\eta}^{*}\left(I-Q_{n}\right)\right\| \leqslant\left\|\left(A_{\eta}^{*}-A^{*}\right)\left(I-Q_{n}\right)\right\|+\left\|U^{*}\left|A^{*}\right|\left(I-Q_{n}\right)\right\| \leqslant\left\|A_{\eta}^{*}-A^{*}\right\|+$ $+\left\|\left|A^{*}\right|\left(I-Q_{n}\right)\right\|$. Оценка (24) вытекает из (9), оценка (25) из свойства $I-Q_{n}=\left(I-Q_{n}\right)^{2}$ неравенства $\left\|A_{\eta}{ }^{*}\left(I-Q_{n}\right)^{2} A_{\eta}\right\| \leqslant\left\|A_{\eta}{ }^{*}\left(I-Q_{n}\right)\right\|^{2}$ и $(24)$.

По (23), (25) имеем $\left\|\left(I-P_{n}\right)\left|A_{\eta}\right|^{2}\right\| \leqslant C(h+\eta)^{2}$, а в силу неравенства моментов также $\|\left(I-P_{n}\right)\left|A_{\eta}\right|^{p \|} \leqslant C(h+\eta)^{p}$ при $p \leqslant 2$. В силу (22) оценка (21) при $p \leqslant 2$ получена, при $p>2$ она вытекает из (23) и из установленной ниже оценки

$$
\left\|A_{\eta}^{*}\left(I-Q_{n}\right) A_{\eta}\left(A_{\eta}^{*} A_{\eta}\right)^{r}\right\| \leqslant C h^{1+\min \left(2 r+1, p_{2}\right)}+C(1+|\ln \eta|) \eta \quad(r \geqslant 0) .
$$

Оценим $\left\|A_{\eta}^{*}\left(I-Q_{n}\right) A_{\eta}\left(A_{\eta}^{*} A_{\eta}\right)^{r}\right\| \leqslant\left\|A_{\eta}^{*}\left(I-Q_{n}\right)\right\|\left\|\left(I-Q_{n}\right) A_{\eta}\left(A_{\eta}^{*} A_{\eta}\right)^{r}\right\|$. В силу равенства $A_{\eta}\left(A_{\eta}{ }^{*} A_{\eta}\right)^{r}=\left(A_{\eta} A_{\eta}{ }^{*}\right)^{r} A_{\eta}$ и полярного разложения $A_{\eta}=\left\|A_{\eta}^{*}\right\| U_{\eta},\left\|U_{\eta}\right\|=1$ имеем $\left\|\left(I-Q_{n}\right) A_{\eta}\left(A_{\eta}^{*} A_{\eta}\right) r\right\| \leqslant\left(I-Q_{n}\right)\left|A_{\eta}^{*}\right|^{2 r+1} \| \leqslant$ $\leqslant\left\|\left(I-Q_{n}\right)\left|A^{*}\right|^{2 r+1}\right\|+\left\|\left|A^{*}\right|^{2 r+1}-\left|A_{\eta}^{*}\right|^{2 r+1}\right\|$. Теперь оценка (26) вытекает из $(24),(9)$ и (14). Лемма доказана.

\section{7. Доказательство теорем $1-3$}

Основные идеи доказательств заимствованы из [ $\left.{ }^{5}\right]$. Доказ а тельство те о ремы 1 . По (4) имеем

$$
\begin{gathered}
u_{n, \alpha}-u_{*}=G_{n, \alpha, \eta}\left(u_{0}-u_{*}\right)-\left[I-g_{\alpha}\left(A_{\eta, n}\right) P_{n} A_{\eta}\left(I-P_{n}\right)\right]\left(I-P_{n}\right) u_{\star}+ \\
+g_{\alpha}\left(A_{\eta, n}\right) P_{n}\left(f_{\delta}-A_{\eta} u_{*}\right), \quad A_{\eta, n}=P_{n} A_{\eta} P_{n}, \quad G_{n, \alpha, \eta}=\left[I-A_{\eta, n} g_{\alpha}\left(A_{\eta, n}\right)\right] P_{n} .
\end{gathered}
$$

При помощи теоремы Банаха-Штейнгауза убеждаемся, что

$$
G_{n, \alpha, \eta}\left(u_{0}-u_{\star}\right) \rightarrow 0 \text { при } n \rightarrow \infty, \quad \alpha \rightarrow 0, \quad \eta \rightarrow 0 .
$$

По условию (3) $\left\|G_{n, \alpha, \eta}\right\| \leqslant C$. Поскольку $u_{*}-$ ближайшее к $u_{0}$ решение уравнения (1), то $u_{0}-u_{\star} \in \mathcal{N}(A) \perp u$, следовательно, $u_{0}-u_{\star} \in \overrightarrow{R(A)}$. Для элементов вида $u=A v(v \in H)$, образующих в $\overline{R(A)}$ плотное подмножество, с учетом полной непрерывности $A$ и условия (3) имеем $\left\|G_{n, \alpha, \eta} u\right\|=\left\|G_{n, \alpha, \eta} P_{n} A v\right\| \leqslant\left\|G_{n, \alpha, \eta}\right\|\left\|P_{n} A\left(I-P_{n}\right)+P_{n}\left(A-A_{\eta}\right) P_{n}\right\|\|v\|+$ $+\left\|G_{n, \alpha, \eta} A_{\eta, n}\right\|\|v\| \leqslant C\left[\left\|A\left(I-P_{n}\right)\right\|+\eta+\left\|G_{n, \alpha, \eta} A_{\eta, n}\right\|\right]\|v\| \rightarrow 0$ при $n \rightarrow \infty$, $\alpha \rightarrow 0 ; \eta \rightarrow 0$.

В силу предположения $p_{1} \geqslant 1 / 2$ по (18) имеем

$$
\begin{gathered}
\left\|\left[I-g_{\alpha}\left(A_{\eta, n}\right) P_{n} A_{\eta}\left(I-P_{n}\right)\right]\left(I-P_{n}\right) u_{*}\right\| \leqslant\left[1+C\left(\eta^{1 / 2}+h^{1 / 2}\right) /\left(\alpha+h_{\eta}\right)^{1 / 2}\right] \times \\
\times\left\|\left(I-P_{n}\right) u_{*}\right\|,
\end{gathered}
$$

где $\left\|\left(I-P_{n}\right) u_{*}\right\| \rightarrow 0$ при $n \rightarrow \infty$, так как $u_{\star} \in \overline{R(A)}$ по условиям $u_{0}, u_{0}-u_{*} \in \overline{R(A)}$.

Из (17) вытекает оценка 


$$
\left\|g_{\alpha}\left(A_{\eta, n}\right) P_{n}\left(f_{\delta}-A_{\eta} u_{*}\right)\right\| \leqslant C \Delta /\left(\alpha+h_{\eta}\right) .
$$

Сходимость $g_{n, \alpha} \rightarrow 0$ при $\Delta \rightarrow 0$ следует из $(27)-(30)$ и из правила выбора $n$ и $\alpha$.

Для оценки ошибки необходимы неравенства

$$
\begin{gathered}
\left\|P_{n} A_{\eta}^{1 / 2} P_{n}-A_{\eta, n}^{1 / 2}\right\| \leqslant C\left\|\left(I-P_{n}\right) A_{\eta}^{1 / 2}\right\|, \\
\left\|A_{\eta}^{r}-A_{\eta, n}^{r}\right\| \leqslant C(h+\eta)^{r} \quad(r \leqslant 1 / 2) .
\end{gathered}
$$

Сценка (31) вытекает с помощью (15) из соотношения $\left\|\left(P_{n} A_{\eta}^{1 / 2} P_{n}\right)^{2}-P_{n} A_{\eta} P_{n}\right\|=\left\|P_{n} A_{\eta}^{1 / 2}\left(P_{n}-I\right) A_{\eta}^{1 / 2} P_{n}\right\| \leqslant\left\|\left(I-P_{n}\right) A_{\eta}^{1 / 2}\right\|^{2}$.

Оденка (32) следует из неравенства $\quad\left\|A_{\eta}^{1 / 2}-A_{\eta, n}^{1 / 2}\right\| \leqslant\left\|A_{\eta}^{1 / 2}-P_{n} A_{\eta}^{1 / 2}\right\|+$ $+\left\|P_{n}\left(A_{\eta}^{1 / 2}-A_{\eta}^{1 / 2} P_{n}\right)\right\|+\left\|P_{n} A_{\eta}^{1 / 2} P_{n}-A_{\eta, n}^{1 / 2}\right\| \leqslant C\left\|\left(I-P_{n}\right) A_{\eta}^{1 / 2}\right\| \quad$ и оценок (15), (16).

Предполагая (6) и используя (8) и (15), можно оценить

$$
\begin{gathered}
\left\|\left(I-P_{n}\right) u_{*}\right\|=\left\|\left(I-P_{n}\right) A^{p}(w-v)\right\| \leqslant\left\|\left(I-P_{n}\right) A^{p}\right\|\|w-v\| \leqslant \\
\quad \leqslant C h^{\min \left(p, p_{1}\right)}, \\
\left\|G_{n, \alpha, \eta}\left(u_{0}-u_{*}\right)\right\|=\left\|G_{n, \alpha, \eta}\left[\left(A^{p}-A_{\eta}^{p}\right)+A_{\eta}^{p}\right] v\right\| \leqslant \\
\leqslant C \eta^{\min (p, 1)}+\left\|G_{n, \alpha, \eta} A_{\eta}^{p}\right\|\|v\| .
\end{gathered}
$$

Рассмотрим сгіерва случай $p \leqslant 1 / 2$. В силу (32), (3) имеем

$$
\begin{gathered}
\left\|G_{n, \alpha, \eta} A_{\eta}^{p}\right\| \leqslant\left\|G_{n, \alpha, \eta}\right\|\left\|A_{\eta}^{p}-A_{\eta, n}^{p}\right\|+\left\|G_{n, \alpha, \eta} A_{\eta, n}^{p}\right\| \leqslant \\
\leqslant C(h+\eta)^{p}+C_{\alpha^{\min \left(p, p_{0}\right)} .}
\end{gathered}
$$

В случае $1 / 2<p<1$ воспользуемся полярным разложением

$$
P_{n} A_{\eta}^{1 / 2}=\left[P_{n} A_{\eta}^{1 / 2}\left(P_{n} A_{\eta}^{1 / 2}\right)^{*}\right]^{1 / 2} U_{\eta, n}=A_{\eta, n}^{1 / 2} U_{\eta, n}, \quad\left\|U_{\eta, n}\right\|=1 .
$$

С учетом равенства $G_{n, \alpha, \eta}=G_{n, \alpha, \eta} P_{n}$ в силу (32), (3) имеем

$\left\|G_{n, \alpha, \eta} A_{\eta}^{p}\right\| \leqslant\left\|G_{n, \alpha, \eta} A_{\eta, n}^{1 / 2}\right\|\left\|U_{\eta, n}\right\|\left\|A_{\eta}^{p-1 / 2}-A_{\eta, n}^{p-1 / 2}\right\|+\left\|G_{n, \alpha, \eta} A_{\eta, n}^{1 / 2} U_{\eta, n} A_{\eta, n}^{p-1 / 2}\right\| \leqslant$

$$
\leqslant C_{\alpha^{\min \left(1 / 2, p_{0}\right)}(h+\eta)^{p-1 / 2}+C \alpha^{\min \left(p, p_{0}\right)} .}
$$

При $p \geqslant 1$ воспользуемся представлением ([p] - целая часть $p)$

$$
P_{n} A_{\eta}^{p}=\sum_{i=1}^{[p]} A_{\eta, n}^{i-1} P_{n} A_{\eta}\left(I-P_{n}\right) A_{\eta}^{p-i}+A_{\eta, n}^{[p]} A_{\eta}^{p-[p]}=: \Sigma+\sigma
$$

вытекающим из равенств $\quad P_{n} A_{\eta}^{p-i}=P_{n} A_{\eta}\left[\left(I-P_{n}\right)+P_{n}\right] A_{\eta}^{p-i-1}$ при $0 \leqslant i \leqslant[p]-1$. В силу (3) и (31) имеем

$$
\begin{gathered}
\left\|G_{n, \alpha, \eta} \Sigma\right\| \leqslant \sum_{i=1}^{[p]}\left\|G_{n, \alpha, \eta} A_{\eta, n}^{i-1}\right\|\left\|\left(I-P_{n}\right) A_{\eta}\right\|\left\|\left(I-P_{\dot{n}}\right) A_{\eta}^{p-i}\right\| \leqslant \\
\leqslant C \sum_{i=1}^{[p]} \alpha^{\min \left(i-1, p_{0}\right)}\left(h^{\min \left(1, p_{1}\right)}+\eta\right)\left(h^{\min \left(p-1, p_{1}\right)}+\eta^{\min (p-1,1)}\right) .
\end{gathered}
$$

В случае $p-[p] \leqslant 1 / 2$ оценим с помощью (32) и (3)

$$
\begin{aligned}
\left\|G_{n, \alpha, \eta} \sigma\right\| & \leqslant\left\|G_{n, \alpha, \eta} A_{\eta, n}^{[p]}\right\|\left\|A_{\eta}^{p-[p]}-A_{\eta, n}^{p-[p]}\right\|+\left\|G_{n, \alpha, \eta} A_{\eta, n}^{p}\right\| \leqslant \\
& \leqslant C \alpha^{\min \left([p], p_{0}\right)}(h+\eta)^{p-[p]}+C \alpha^{\min \left(p, p_{0}\right)} .
\end{aligned}
$$

В случае $p-[p]>1 / 2$ с учетом равенств $A_{\eta, n}^{[p]}=A_{\eta, n}^{[p]} P_{n}$

оценок (32) и (3) имеем 


$$
\begin{aligned}
\left\|G_{n, \alpha, \eta} \sigma\right\| & =\left\|G_{n, \alpha, \eta} A_{\eta, n}^{[p]} P_{n} A_{\eta}^{1 / 2} A_{\eta}^{p-[p]-1 / 2}\right\| \leqslant \\
& \leqslant\left\|G_{n, \alpha, \eta} A_{\eta, n}^{[p]+1 / 2}\right\|\left\|U_{\eta, n}\right\|\left\|A_{\eta}^{p-[p]-1 / 2}-A_{\eta, n}^{p-[p]-1 / 2}\right\|+ \\
& +\left\|G_{n, \alpha, \eta} A_{\eta, n}^{[p]+1 / 2} U_{\eta, n} A_{\eta, n}^{p-[p]-1 / 2}\right\| \leqslant \\
& \leqslant C \alpha^{\min \left([p]+1 / 2, p_{0}\right)}(h+\eta)^{p-[p]-1 / 2}+C \alpha^{\min \left(p, p_{0}\right)} .
\end{aligned}
$$

Используя неравенства типа $x^{\Theta} y^{1-\Theta} \leqslant \max (x, y) \quad(0<\Theta<1)$, $(x+y)^{r} \leqslant 2^{r} \max \left(x^{r}, y^{r}\right)$, по (27), (29), (30), (33)-(35), (37)-(41) имеем

$$
\left\|u_{n, \alpha}-u_{*}\right\| \leqslant C\left[h^{\min \left(p, p_{1}\right)}+\alpha^{\min \left(p, p_{0}\right)}+\eta^{\min (p, 1)}+\Delta /\left(h_{\eta}+\alpha\right)\right] .
$$

Отсюда и из правил выбора $n$ и $\alpha$ вытекает оценка (7).

Теорема 1 доказана.

Доказательство те оремы 2 аналогично доказательству теоремы 1, вместо леммы 3 используется лемма 4.

Доказ а тельство те оремы 3 . По (5) имеем

$u_{n, \alpha}-u_{\star}=K_{n, \alpha, \eta}\left(u_{0}-u_{*}\right)-\left(I-P_{n}\right) u_{\star}+g_{\alpha}\left(B_{\eta, n}\right) A_{\eta, n}^{* 4}\left(f_{\delta}-A_{\eta} u_{\star}\right)$,

где $\quad A_{\eta, n}=Q_{n} A_{\eta}, \quad B_{\eta, n}=A_{\eta}^{*} Q_{n} A_{\eta}, \quad K_{n, \alpha, \eta}=\left[I-B_{\eta, n} g_{\alpha}\left(B_{\eta, n}\right)\right] P_{n}$.

Мы учли, что в методе наименьшей ошибки $P_{n} A_{\eta}{ }^{*} Q_{n}=A_{\eta}{ }^{*} Q_{n}$, откуда $Q_{n} A_{\eta} P_{n}=Q_{n} A_{\eta}$ и, следовательно, $\quad A_{\eta, n}^{*} A_{\eta}\left(I-P_{n}\right)=A_{\eta}^{*} Q_{n} A_{\eta} P_{n} \times$ $X\left(I-P_{n}\right)=0$.

С помощью теоремы Банаха-Штейнгауза убеждаемся, что

$$
K_{n, \alpha, \eta}\left(u_{0}-u_{*}\right) \rightarrow 0 \quad \text { при } \quad n \rightarrow \infty, \quad \alpha \rightarrow 0, \quad \eta \rightarrow 0 .
$$

По условию (3) $\left\|K_{n, \alpha, \eta}\right\| \leqslant C ; u_{0}-u_{*} \in \overline{R\left(A^{*}\right)} ;$ для элементов $u=$ $=A^{*} A v(v \in H)$, образующих в $\overline{R\left(A^{*}\right)}$ плотное подмножество, имеем по (3)

$$
\begin{gathered}
\left\|K_{n, \alpha, \eta} u\right\|=\left\|K_{n, \alpha, \eta} A^{*} A v\right\| \leqslant\left\|K_{n, \alpha, \eta}\right\| \| A^{*}\left(I-Q_{n}\right) A+A^{*} Q_{n}\left(A-A_{\eta}\right)+ \\
+\left(A^{*}-A_{\eta}\right) Q_{n} A_{\eta}\|\| v\|+\| K_{n, \alpha, \eta} B_{\eta, n}\|\| v \| \leqslant \\
\leqslant C\left[\left\|\left(I-Q_{n}\right) A\right\|^{2}+\eta+\left\|K_{n, \alpha, \eta} B_{\eta, n}\right\|\right] \rightarrow 0 \\
\text { при } n \rightarrow \infty, \quad \alpha \rightarrow 0, \quad \eta \rightarrow 0 .
\end{gathered}
$$

С учетом $u_{*} \in \overline{R\left(A^{*}\right)}$ имеем

$$
\left(I-P_{n}\right) u_{*} \rightarrow 0 \text { при } n \rightarrow \infty .
$$

Из тождества $f_{\delta}-A_{\eta} u_{*}=\left(f_{\delta}-f\right)+\left(A-A_{\eta}\right) u_{*}$, равенства $A_{\eta, n}^{*}=A_{\eta, n}^{*} Q_{n} \quad$ и неравенства (19) вытекает оценка

$$
\left\|g_{\alpha}\left(B_{\eta, n}\right) A_{\eta, n}^{*}\left(f_{\delta}-A_{\eta} u_{*}\right)\right\| \leqslant C \Delta /\left(\alpha^{1 / 2}+h_{\eta}\right) .
$$

Сходимость $e_{n, \alpha} \rightarrow 0$ при $\Delta \rightarrow 0$ следует из $(42)-(45)$ и из правила выбора $n$ и $\alpha$.

Пусть выполнено условие (6), а также (9) с $p_{2} \geqslant 1$. По (21) и (14) имеем

$$
\begin{gathered}
\left\|\left(I-P_{n}\right) u_{*}\right\|=\left\|\left(I-P_{n}\right)|A|^{p}(w-v)\right\| \leqslant \\
\leqslant C h^{1+\min \left(p-1, p_{2}\right)}+C(1+|\ln \eta|) \eta^{\min (p, 1)},
\end{gathered}
$$




$$
\begin{gathered}
\left\|K_{n, \alpha, \eta}\left(u_{0}-u_{*}\right)\right\|=\left\|K_{n, \alpha, \eta}\left[\left(|A|^{p}-\left|A_{\eta}\right|^{p}\right)+\left|A_{\eta}\right|^{p}\right] v\right\| \leqslant \\
\leqslant C(1+|\ln \eta|) \eta^{\min (p, 1)}+\left\|K_{n, \alpha, \eta}\left|A_{\eta}\right|^{p}\right\|\|v\| .
\end{gathered}
$$

Из (25) и (15) вытекает оценка

$$
\left\|\left|A_{\eta}\right|^{r}-B_{\eta, n}^{r / 2}\right\| \leqslant C(h+\eta)^{r} \quad(r \leqslant 2) .
$$

При $p \leqslant 2$ по (48) и (3) имеем

$$
\begin{aligned}
\left\|K_{n, \alpha, \eta}\left|A_{\eta}\right|^{p}\right\| & \leqslant\left\|K_{n, \alpha, \eta}\right\|\left\|\left|A_{\eta}\right|^{p}-B_{\eta, n}^{p / 2}\right\|+\left\|K_{n, \alpha, \eta} B_{\eta, n}^{p / 2}\right\| \leqslant \\
& \leqslant C(h+\eta)^{p}+C \alpha^{\min \left(p / 2, p_{0}\right)} .
\end{aligned}
$$

При $p>2$ воспользуемся представлением

$$
\begin{aligned}
\left|A_{\eta}\right|^{p} & =B_{\eta, n}^{p / 2}+B_{\eta, n}^{[p / 2]}\left[\left|A_{\eta}\right|^{p-2[p / 2]}-B_{\eta, n}^{p / 2-[p / 2]}\right]+ \\
& +\sum_{i=1}^{[p / 2]} B_{\eta, n}^{i-1} A_{\eta}^{*}\left(I-Q_{n}\right) A_{\eta}\left|A_{\eta}\right|^{p-2 i},
\end{aligned}
$$

получаемым привлечением равенств

$\times A_{\eta}\left|A_{\eta}\right|^{p-2 i-2}$ при $0 \leqslant i \leqslant[p / 2]-1$.

$$
\left|A_{\eta}\right|^{p-2 i}=A_{\eta}^{*}\left[\left(I-Q_{n}\right)+Q_{n}\right] \times
$$

По (3), (48) и (26) имеем

$$
\begin{aligned}
\left\|K_{n, \alpha, \eta}\left|A_{\eta}\right|^{p}\right\| & \leqslant\left\|K_{n, \alpha, \eta} B_{, \eta, n}^{p / 2}\right\|+\left\|K_{n, \alpha, \eta} B_{\eta, n}^{[p / 2]}\right\|\left\|\left|A_{\eta}\right|^{p-2[p / 2]}-B_{\eta, n}^{p / 2-[p / 2]}\right\|+ \\
& +\sum_{i=1}^{[p / 2]}\left\|K_{n, \alpha, \eta} B_{\eta, n}^{i-1}\right\| !\left\|A_{\eta}^{*}\left(I-Q_{n}\right) A_{\eta}\left|A_{\eta}\right|^{p-2 i}\right\| \leqslant \\
& \leqslant C \alpha^{\min \left(p / 2, p_{0}\right)}+C \alpha^{\min \left([p / 2], p_{0}\right)}(h+\eta)^{p-2[p / 2]}+ \\
& +C \sum_{i=1}^{[p / 2]} \alpha^{\min \left(i-1, p_{0}\right)}\left[h^{1+\min \left(p-2 i+1, p_{2}\right)}+(1+|\ln \eta|) \eta\right] .
\end{aligned}
$$

По $(42),(45)-(47),(49)$ и $(50)$ имеем

$$
\begin{aligned}
\left\|\mathrm{u}_{n, \alpha}-u_{*}\right\| & \leqslant C\left[h^{1+\min \left(p-1, p_{2}\right)}+\alpha^{\min \left(p / 2, p_{0}\right)}+\right. \\
& \left.+(1+|\ln \eta|) \eta^{\min (p, 1)}+\Delta /\left(h_{\eta}+\alpha^{1 / 2}\right)\right] .
\end{aligned}
$$

Отсюда и из правил выбора $n$ и $\alpha$ вытекает оценка (7).

Теорема 3 доказана.

Основные результаты настоящей статьи анонсированы в $\left[{ }^{28}\right]$.

В заключение автор выражает глубокую благодарность Г. Вайникко за постановку задачи и многие полезные советы.

\section{ЛИТ Е РАТ У РА}

1. Тихонов А. Н., Арсенин В. Я. Методы решения некорректных задач. М., «Наука», 1974.

2. Иванов В. К., Васин В. В., Танана В. П. Теория линейных некорректных задач и ее приложения. М., «Наука», 1978.

3. Лисковец О. А. Вариационные методы решения неустойчивых задач. Минск, «Наука и техника», 1981.

4. Танана В. П. Методы решения операторных уравнений. М., «Наука», 1981.

5. Вайникко $\Gamma$. Методы решення линейных некорректно поставленных задач в гильбертовых пространствах. Тарту, Изд. ТГУ, 1982.

6. Лисковец $O$. А. Итоги науки и техники (матем. анализ). М., Изд. ВИНИТИ, 20, $116-178$ (1982).

7. Natterer, F. RAIRO Analyse numerique, 11, № 3, 271-278 (1977).

8. Кнопова С. М., Савёлова Т. И. Ж. вычисл. матем. и матем. физ., 19, № 5, $1091-1096$ (1979).

9. Нванов В. В., Кудринский В. Ю. Ж. вычисл. матем. и матем. физ., 6, № 5, 831-841 (1966).

10. Калякин Л. А. В кн.: Методы решения условно-корректных задач. Свердловск, $1975,53-66$. 
11. Marti, J. T. Math. Comput., 34, № 150, 521-527 (1980).

12. Groetsch, C. W. J. Integr. Equat., 4, № 2, 173-182 (1982).

13. Hickey, K. R., Luecke, G. R. SIAM J. Numer. Anal., 19, № 3, 623-628 (1982).

14. Вайникко $\Gamma$. В кн.: Тезисы конференции «Методы алгебры и анализа». Тарту, Изд. ТГУ, 1983, 106-109.

15. Страхов В. Н. Ж. вычисл, матем. и матем. физ., 10, № 1, 204-210, (1970).

16. Савёлова Т. И. Ж. вычисл. матем. и матем. физ., 14, № 4, 1027-1031 (1974).

17. Nashed, M. Z., Wahba, G. Math. Comput., 28, № 125, 69-80 (1974).

18. Бушманова М. В. Изв. вузов. Математика, № 9, 11-17 (1977).

19. Natterer, F. Numer. Math., 28, № 3, 329-341 (1977).

20. Richter, G. R. SIAM J. Numer. Anal., 15, № 3, 511-522 (1978).

21. Воронин В. В., Цецохо В. А. Ж. вычисл. матем. и матем. физ., 21, № 1, 40-53 (1981).

22. Seidman T. I. Contr. and Cybern., 10, № 1-2, 31-71 (1981).

23. Хямарик У. А. Тр. вычисл, центра ТГУ, 50, 69-90 (1983).

24. Baker, C. T. H. The Numerical Treatment of Integral Equations. Oxford, Clarendon Press, 1977.

25. Brunner, H. J. Comput. and Appl. Math., 8, № 3, 213-229 (1982).

26. Апарцин A. C. В кн.: Методы оптимизации и их приложения. Новосибирск, «Наука», $1982,138-146$.

27. Красносельский М. А., Вайникко Г. М., Забрейко П. П., Рутицкий Я. Б., Стеценко В. Я. Прнолиженное решение операторных уравнений. М., «Наука», 1969.

28. Хямарик У. А. В кн.: Тезисы конференции «Методы алгебры и анализа», Тарту, Изд. ТГУ, 1983, 145-147.

Тартуский

государственнний университет
Поступила в редакцию 29/IX 1983

\section{U. HAMARIK}

\section{REGULARISEERITUD PROJEKTSIOONIMEETODID MITTEKORREKTSETE OLESANNETE LAHENDAMISEKS}

Artiklis on vaadeldud mittekorrektse ülesande kaheparameetrilist regularisatsiooni: ülesanne diskretiseeritakse projektsioonimeetodil ja seejärel regulariseeritakse. Projektsioonimeetodit on rakendatud tingimustel, kui projekteeritud ülesande mōõdete arv on vaadeldav ühe regularisatsiooniparameetrina. On toodud parameetrite valiku eeskirjad nii regulariseeriva algoritmi kui ka optimaalse järguga veahinnangu saamiseks.

\section{U. HAMARIK}

\section{REGULARIZED PROJECTION METHODS FOR ILL-POSED PROBLEMS}

Let $H, F$ be Hilbert spaces, $A \in L(H, F)$ be a compact operator. Consider problem

$$
A u=f ;
$$

$N(A)$ need not be trivial, $f \in R(A)$. Instead of $A$ and $f$ are given $A_{\eta} \in L(H, F)$ and $f_{o}$ with errors $\left\|A_{\eta}-A\right\| \leqslant \eta,\left\|f_{\delta}-f\right\| \leqslant \delta$. Problem (1) is discretized by the projection method, using orthogonal projections $P_{n}: H \rightarrow H_{n}, Q_{n}: F \rightarrow F_{n}, \operatorname{dim} H_{n}=\operatorname{dim} F_{n}=n$. Discretized problem $Q_{n} A_{\eta} P_{n} u_{n}=Q_{n} f_{\delta}, u_{n} \in H_{n}$ is regularized by a method of class of [5], including methods of Tikhonov and Lavrentyev, iteration methods, and others. We, namely, use function $g_{\alpha}$ with properties (2), (3) and approximate nearest to initial approximation $u_{0}$ least square solution of (1) by $u_{n, \alpha}$ in (4) (selfadjoint case) or (5).

Theorem 1. Let $F=H, A=A^{*} \geqslant 0, A_{\eta}=A_{\eta}{ }^{*} \geqslant 0$. Discretize (1) by method of Galerkin $\left(F_{n}=H_{n}\right)$. Let $H_{n} \cap N(A)=0, \quad P_{n} u \rightarrow u$ if $n \rightarrow \infty \quad \mathrm{V} u \in R \overline{R\left(A^{*}\right)}$ and in (8) let $p_{1} \geqslant 1 / 2$. Choose $n$ and $\alpha$ in (4) according to $\Delta=\max (\delta, \eta)$, so that $n \rightarrow \infty, \alpha \rightarrow 0$, $\Delta /(h+\alpha) \rightarrow 0$ if $\Delta \rightarrow 0$. (Here $\left.h=\inf \left\{\left(A u_{n}, u_{n}\right) /\left\|u_{n}\right\|^{2}, \quad u_{n} \in H_{n}\right\}\right)$. Then $e_{n, \alpha}:=$ $=\left\|u_{n, \alpha}-u_{*}\right\| \rightarrow 0$ if $\Delta \rightarrow 0$. In assumption (6) error estimate of optimal order (7) holds by choosing $n$ and $\alpha$ according to rules 1)-3) in Theor. 1 .

Analogous theorems are also proved for the least square method $\left(F_{n}=A_{\eta} H_{n}\right)$ and for the method of minimal error $\left(H_{n}=A_{\eta}{ }^{*} F_{n}\right)$. Condition number bounds for the coefficient matrix in (4) or (5) are obtained. Applications to Volterra integral equation of the first kind are given. 